\title{
c-Myc and EBV-LMPI: two opposing regulators of the HLA class I antigen presentation machinery in epithelial cells
}

\begin{abstract}
CS Tudor*, , CW Dawson ${ }^{2}$, J Eckhardt ${ }^{3}$, G Niedobitek ${ }^{4}$, AC Büttner ${ }^{5}$, B Seliger6, A Hartmann' and M Buettner'
'Institute of Pathology, Department of Nephropathology, Friedrich-Alexander-University, Erlangen-Nuremberg, Krankenhausstr. 8-10, 91054 Erlangen, Germany; ${ }^{2}$ Cancer Research UK, Birmingham Cancer Centre, University of Birmingham, Edgbaston, Birmingham BI 5 T22, UK; ${ }^{3}$ Department of Immune Modulation at the Department of Dermatology, University Hospital Erlangen, Hartmannstr. 14,91052 Erlangen, Germany; ${ }^{4}$ Institutes of Pathology, Sana Klinikum Lichtenberg/Unfallkrankenhaus Berlin, Fanningerstr. 32, 10365 Berlin/Warener Str. 7, I 2683 Berlin, Germany; ${ }^{5}$ School of Psychology, University of Birmingham, Edgbaston, Birmingham B 5 2TT, UK; ' Institute of Medical Immunology, Martin Luther University Halle-Wittenberg, Magdeburger Str. 2, 06097 Halle (Saale), Germany
\end{abstract}

BACKGROUND: Epstein-Barr virus (EBV)-encoded latent membrane protein I (LMPI) up-regulates the human leukocyte antigen (HLA) class I antigen presentation machinery (APM). This appears counterintuitive with immune evasion in EBV-associated tumours like nasopharyngeal carcinoma (NPC).

METHODS: Latent membrane protein I-transfected epithelial cell lines were used as a model system to study the impact of LMPI and c-Myc on HLA class I components. The expression of components of the HLA class I APM, c-Myc and Ki-67 was analysed in LMPI + and LMPI - NPC by immunohistochemistry.

RESULTS: In epithelial cells, LMPI up-regulated HLA class I APM. This effect could be counteracted by c-Myc, which itself was up-regulated by LMPI apparently through IL6 induction and Jak3/STAT3 activation. Studies of NPC biopsies revealed down-regulation of HLA class I APM expression. No difference was observed between LMPI + and LMPI - NPC. However, expression of Ki-67 and c-Myc were up-regulated in LMPI + tumours.

CONCLUSION: These findings raise the possibility that c-Myc activation in NPC might antagonise the effect of LMPI on HLA class I expression thus contributing to immune escape of tumour cells.

British Journal of Cancer (2012) I 06, 1980-1988. doi:I0.1038/bjc.2012.197 www.bjcancer.com

Published online 15 May 2012

(C) 2012 Cancer Research UK

Keywords: HLA class I; LMPI; EBV; NPC

More than $90 \%$ of the world population are Epstein-Barr virus (EBV) carriers. Although EBV leads mostly to an asymptomatic infection in childhood, there is sufficient evidence for its carcinogenicity in the causation of lymphomas and epithelial cancers, such as gastric and nasopharyngeal carcinoma (NPC). In addition to tumour antigens, EBV-associated tumours express viral antigens that are potential targets for an anti-tumoural immunity (Young and Rickinson, 2004). However, the fact that they are not eliminated by the immune system suggests that EBVpositive tumour cells establish mechanisms to evade immune attack. One potential immune evasion mechanism is the downregulation of components of the human leukocyte antigen (HLA) class I antigen presentation machinery (APM), which may allow tumour cells to evade cytotoxic T lymphocyte (CTL) attack (Seliger et al, 2002; Chang et al, 2003). Even partial HLA class I APM downregulation promoted disease progression in several malignancies including NPC (Ogino et al, 2003, 2007; Demanet et al, 2004; Vitale et al, 2005). Interestingly, however, it has also been reported that the down-regulation of HLA class I makes tumour cells more sensible to natural killer (NK) cell attack (Ravetch and

*Correspondence: Dr CS Tudor; E-mail: Silke.Tudor@uk-erlangen.de Received 3 February 2012; revised 13 April 2012; accepted 17 April 2012; published online 15 May 2012
Lanier, 2000), so that the loss of HLA class I might be a disadvantage for the tumour.

Antigen presentation through HLA class I proteins involves antigen processing into peptides in the proteasome and transport into the endoplasmic reticulum (ER) via a transmembranetransporter associated with antigen processing (TAP). In the ER, the peptides are loaded onto a complex of the HLA class I heavy chain (HC) and $\beta_{2}$-microglobuline $\left(\beta_{2}-\mathrm{m}\right)$ with the assistance of chaperones including tapasin. Thereafter, the complex is translocated via the trans-Golgi to the cell surface for antigen presentation (Flutter and Gao, 2004).

The EBV-encoded latent membrane protein (LMP)1, however, has been shown to induce the expression of HLA class I, TAP1 and TAP2 in B cells (Rowe et al, 1995) and of HLA class I in epithelial cells (Murray et al, 1998) inducing an immunogenic phenotype. This appears counterintuitive with immune evasion with regard to CTL attack. Latent membrane protein 1 acts as a constitutively active member of the tumour necrosis factor receptor family (CD40) (Uchida et al, 1999), activating a multitude of intracellular signalling pathways in a ligand-independent manner. Particularly, the STAT3 pathway can be strongly activated by LMP1 via JAK3 kinase (Eliopoulos et al, 1999; Gires et al, 1999; Chen et al, 2003) or an increase of IL6 secretion (Eliopoulos et al, 1997; Hirano et al, 2000). Signal transducer and activator of transcription 3, in turn, upregulates c-myc (Bromberg et al, 1999; Bowman et al, 2001), which 
has been shown to down-regulate HLA class I APM expression in human melanoma cell lines (Versteeg et al, 1988; Blom et al, 1997), several carcinoma cell lines (Ottesen et al, 1990; Belldegrun et al, 1993) and in B cells (Staege et al, 2002). Therefore, one could hypothesise an auto-regulatory loop involving LMP1, STAT3 and c-myc to compensate the immunogenicity of LMP1.

The expression of LMP1 in epithelial tumours is both variable and heterogenous. Whereas LMP1 can be detected at the RNA level in the vast majority of NPC cases (Lin et al, 2001), and LMP1 specific antibodies are found in sera of $>70 \%$ of patients (Xu et al, 2000), immunohistochemical staining of LMP1 protein is more variable, ranging between $20 \%$ and $60 \%$ of cases (Fahraeus et al, 1988; Niedobitek et al, 1992). Previous investigations on the expression of HLA class I APM components in NPC yielded conflicting results (Lai et al, 1990; Khanna et al, 1998; Sengupta et al, 2006; Ogino et al, 2007). Interestingly, no differences were observed when comparing LMP1 + and LMP1 - NPC cases (Ogino et al, 2007). The present study was designed to investigate the interplay of LMP1, STAT3 and c-Myc in the regulation of HLA class I APM in epithelial cells and to test whether those mechanisms could be operational in an EBV-associated carcinoma using the example of NPC.

A

EBV/LMP-1

HLA-A

$\beta_{2}-m$ TAP1

LMP2
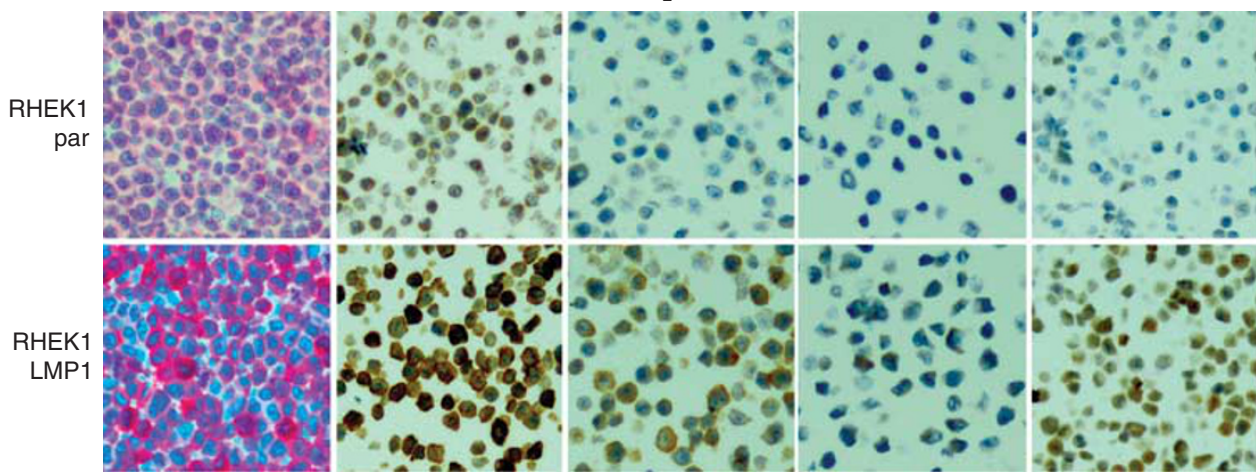

B

RHEK1
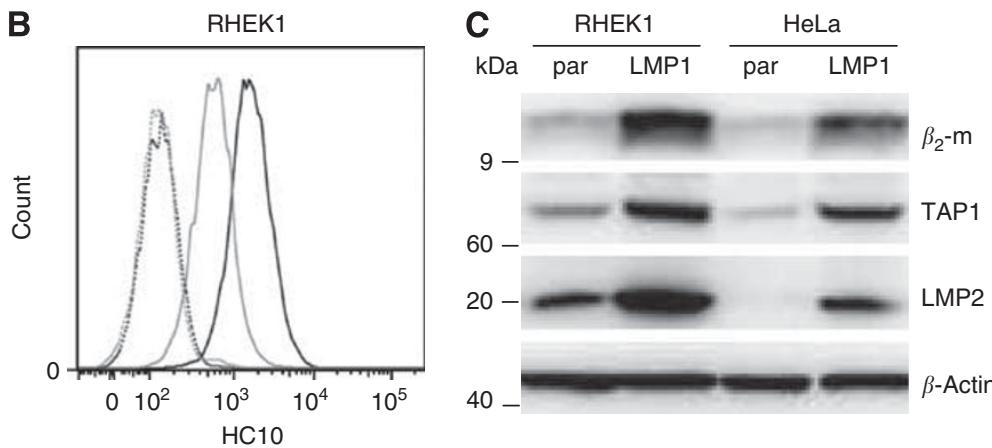

D
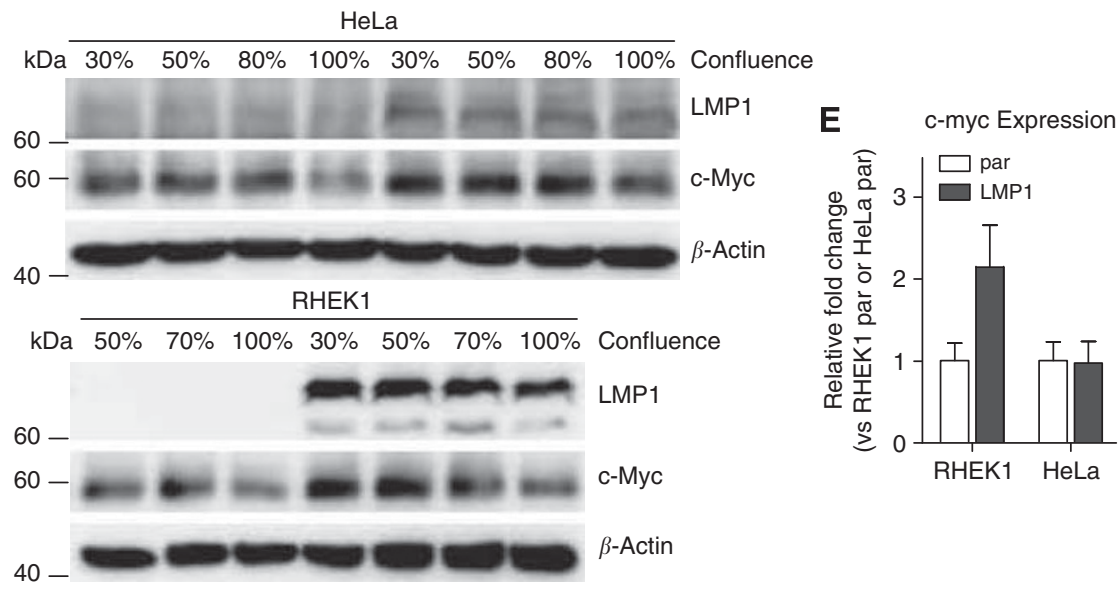

Figure I Induction of HLA class I APM components and of c-Myc in stably LMPI-transfected epithelial cell lines. (A) Comparative immunocytochemistry of RHEKI and RHEKI/LMPI cell lines. HLA-A, $\beta_{2}-\mathrm{m}$, TAPI and LMP2 were visibly up-regulated in response to LMPI expression. One representative experiment of 3. (B) Flow cytometric analysis of RHEKI and RHEKI/LMPI showing the induction of the HLA class I HC (HCIO) in LMPI expressing cells. Dotted/solid light and dark lines represent the control staining without primary antibody/HCIO expression in RHEKI and RHEKI/LMPI, respectively. One representative experiment of 3. (C) Western blot analysis of RHEKI and HeLa cells. Up-regulation of $\beta_{2}-\mathrm{m}$, TAPI and LMP2 in stable LMPI-transfected cell lines. One representative experiment of 3. (D) Western blot analysis of c-Myc expression depending on the confluence of the cell culture. The amount of c-Myc protein decreases with increasing confluence. When comparing RHEKI and HeLa parental with RHEKI/LMPI and HeLa/LMPI at 50\% confluence, an increase of c-Myc is seen in the LMPI-transfected cell line. One representative experiment of 3. (E) qRT-PCR analysis of RHEKI, RHEKI/LMPI, HeLa and HeLa/LMPI cells for c-myc expression. Latent membrane protein I induced c-myc expression in RHEKI cells, whereas no effect in HeLa cells on RNA level was observed. 


\section{METHODS}

\section{Tissues and cell lines}

RHEK1 and HeLa and the stably LMP1 (type B95.8) transfected RHEK1/LMP1 and HeLa/LMP1 cell lines (Siegler et al, 2004; Dawson et al, 2008) were maintained at $37^{\circ} \mathrm{C}$ and $5 \% \mathrm{CO}_{2}$ in RPMI-1640 + GlutaMAX supplemented with $10 \%$ fetal bovine serum, $50 \mathrm{U} \mathrm{ml}^{-1}$ penicillin and $50 \mu \mathrm{g} \mathrm{ml}^{-1}$ streptomycin (Invitrogen, Darmstadt, Germany). Paraffin blocks of RHEK1 and RHEK1/LMP1 cells were generated using a commercially available Cell Block Preparation System (Shandon Cytoblock, Thermo Scientific, Astmoor, UK). The cell pellets were embedded in paraffin and transferred into tissue microarrays (TMA). Formalin-fixed and paraffin-embedded specimens of columnar epithelium $(n=17)$ and metaplastic squamous epithelium $(n=10)$ of the nasopharynx were retrieved from the files of the Institute of Pathology, University Erlangen and served as control tissue. In all, 16 biopsies of EBV-associated NPC have been described earlier (Heussinger et al, 2004; Buettner et al, 2006) or were retrieved from the files of the Institute of Pathology, University Erlangen and characterised by EBER in situ hybridisation and LMP1 immunohistochemistry as described earlier (Niedobitek and Herbst, 2001; Buettner et al, 2006). The control tissues and 12 of the NPC were investigated in TMA with cores of $2 \mathrm{~mm}$ diameter as described (Kasper et al, 2005; van Oers et al, 2007). Four NPC complete sections instead of TMA sections were investigated, in which the markers of the HLA class I APM, c-Myc and Ki-67 showed a diffuse staining pattern. Tissue biopsies were used in accordance with a statement of our local ethical board.

\section{Immunohistochemistry}

Sections $(2 \mu \mathrm{m})$ were deparaffinised in xylene and rehydrated in graded ethanol (100-70\%). For the detection of components of the HLA class I APM HLA-A and HC detected by HC10 mouse monoclonal antibodies $(\mathrm{mAb})$ were applied, which were formerly described (Lampson et al, 1983; Stam et al, 1986; Sernee et al, 1998; Ogino et al, 2003; Perosa et al, 2003; Bandoh et al, 2005; Wang et al, 2005) (Supplementary Table S1). Antigen retrieval was performed as indicated in Supplementary Table S1. Endogenous peroxidase was blocked with Peroxidase-Block (Dako Cytomation, Hamburg, Germany). Primary antibodies were incubated on the slides for $30 \mathrm{~min}$ at room temperature (RT). An HRP-based EnVision + Kit (Dako Cytomation) was used for detection and $\mathrm{DAB}$ (Dako Cytomation) served as a chromogen. For the detection of c-Myc, Ki-67, HLA-B and EBV/LMP1 antigen retrieval was performed as indicated in Supplementary Table S1. Primary antibodies were added overnight at RT. Subsequently, a biotinlabelled polyclonal goat anti-rabbit secondary antibody $(1: 100$, Dako Cytomation) was added for $30 \mathrm{~min}$ at RT followed by an
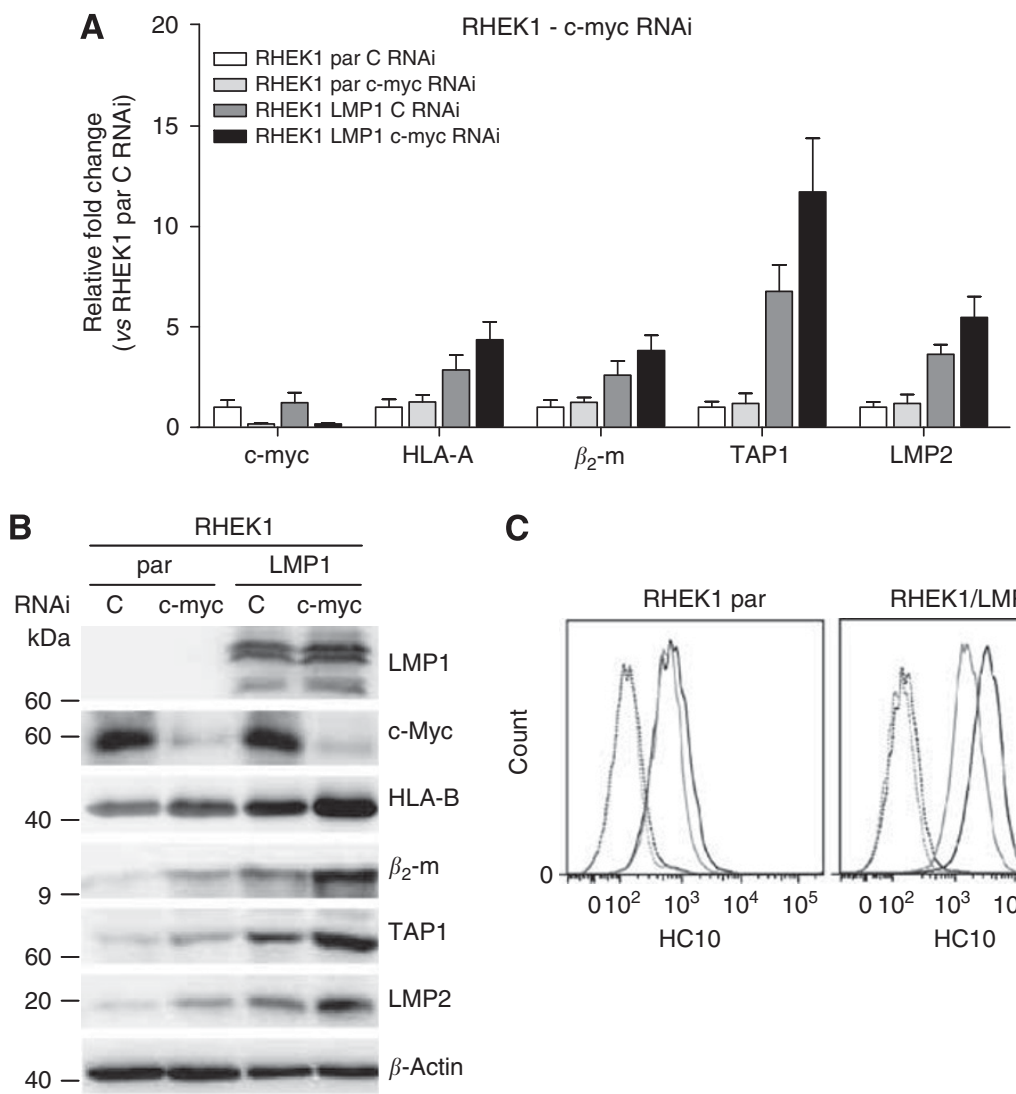

C

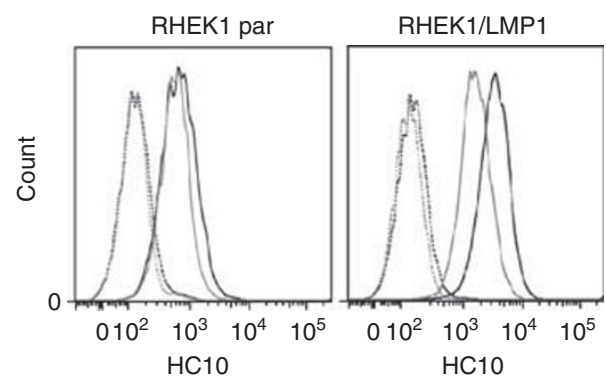

Figure 2 Inhibitory effect of c-myc on the expression of HLA class I APM components in epithelial cells. $(\mathbf{A})$ qRT-PCR analysis $\left(\Delta \Delta C_{T}\right.$ method with efficiency correction) of RHEKI cells for HLA class I APM components after treatment with c-myc siRNA. c-myc knockdown stimulated gene expression of HLA-A, $\beta_{2}-\mathrm{m}$, TAPI and LMP2 in both RHEKI parental and RHEKI/LMPI cells. Latent membrane protein I expression enhanced the c-myc knockdown effect in RHEKI cells. Data of three independent experiments are shown with standard deviations. (B) Western blot analysis of RHEKI parental and RHEKI/LMPI after knockdown of c-myc with siRNA. The expression of c-Myc protein is almost totally abolished. After c-myc knockdown, an up-regulation of HLA-B, $\beta_{2}-\mathrm{m}$, TAPI and LMP2 is observed in RHEKI parental and RHEKI/LMPI. One representative experiment of 2. (C) Flow cytometric analysis of RHEKI parental and RHEKI/LMPI after knockdown of c-myc with siRNA. An up-regulation of HLA class I HC (HCIO) is seen in parental cells and RHEKI/LMPI. The effect of LMPI on the expression of $\mathrm{HCIO}$ is enhanced after c-myc knockdown. Dotted/solid light and dark lines represent the control staining without primary antibody/HClO expression for control and c-myc knocked down cells, respectively. One representative experiment of 3. 
incubation with streptavidin-biotinylated horseradish peroxidase complex (Dako Cytomation). Then tyramide signal amplification was performed as described previously (Greiner et al, 2005) or an alkaline phosphatase-labelled polymer kit was added (ZytochemPlus AP-PolymerKit, Zytomed Systems, Berlin, Germany). Aminoethyl carbazole (Zymed Laboratories, Berlin, Germany) or Fast Red (Sigma-Aldrich, Deisenhof, Germany) served as chromogens. Sections were counterstained with Mayer's Hemalaun solution (Merck, Darmstadt, Germany).

\section{Semi-quantitative evaluation of immunohistochemical and immunocytochemical stainings}

A similar scoring system has been used for the HLA class I APMspecific antibodies before (Seliger et al, 2010). Briefly, the percentage of positive tumour cells was scored as negative $(0),<25 \%$ positive (1), $25-50 \%$ positive $(2)$ or $>50 \%$ positive (3) and the staining intensity was scored $(0=$ negative, $1=$ weak, $2=$ moderate, $3=$ strong). The combined score was obtained by adding both values. For the quantification of the c-Myc and Ki-67 expression, the percentage of tumour or epithelial cells with positive nuclear signal was evaluated.

\section{Statistical analysis}

For comparisons of the semi-quantitative immunohistochemical stainings, Mann-Whitney tests were used for ordinal data and for data with a skewed distribution. Comparisons were made between NPC and controls, and between LMP1 + and LMP1 - samples using SPSS17.0 bioinformatics software (SPSS Inc., Chicago, IL, USA). $P$-values $<0.05$ were accepted as statistically significant. When directional hypotheses were tested, one-tailed $P$-values were used.

\section{Western blotting}

For western blot analysis, monoclonal mouse anti-LMP1 $(1: 200$, CS.1-4, Dako Cytomation), polyclonal rabbit anti-TAP1 (1:500, Stressgen, Victoria, Canada), monoclonal rabbit anti-c-Myc $(1: 1000$, clone Y69, Abcam, Cambridge, UK), polyclonal rabbit anti- $\beta_{2}$-m (1:500, Dako Cytomation), polyclonal rabbit anti-LMP2 $(1: 1000$, Abcam) and monoclonal rabbit anti-HLA-B $(1: 1000$, Abcam) were used. In all, 50\% confluent cells were lysed in RIPA buffer and sonicated. For cytoplasmic and nuclear fractionation, cells were incubated in cytoplasmic extraction buffer $(10 \mathrm{~mm}$ Tris, $\mathrm{pH} 7.5 ; 40 \mathrm{~mm} \mathrm{KCl} ; 2 \mathrm{~mm} \mathrm{MgCl}$; $10 \%$ glycerol; $0.125 \% \mathrm{NP} 40 ; 1 \mathrm{~mm}$ PMSF; protease inhibitor cocktail (Roche, Mannheim, Germany)) for $5 \mathrm{~min}$ on ice and centrifuged. Pellets were resuspended in $0.25 \mathrm{M}$ sucrose buffer, layered on a $0.35-\mathrm{M}$ sucrose fraction and centrifuged. The pellet was resuspended in nuclear lysis buffer $(10 \mathrm{~mm}$ Hepes; $\mathrm{pH} \quad 7.5 ; 500 \mathrm{~mm} \mathrm{NaCl} ; 1 \%$ Triton X-100; $10 \%$ glycerol; $1 \mathrm{~mm}$ PMSF; protease inhibitor cocktail (Roche)) and sonicated. After heat-denaturation proteins were separated by sodium dodecyl sulphate-polyacrylamide gel electrophoresis.

\section{qRT-PCR}

Total RNA was extracted using an RNeasy kit (RNeasy kit, Qiagen, Hilden, Germany). Complementary DNA was generated by using random hexamers and RevertAid reverse transcriptase (Fermentas, Leon-Rot, Germany), applying $500 \mathrm{ng}$ RNA. Primers used to quantify gene transcripts by qRT-PCR are summarised in Supplementary Table S2 (Dahl et al, 2007; Diosdado et al, 2009; Respa et al, 2011). Glyceraldehyde 3-phosphate dehydrogenase was stably expressed in control and treated samples and therefore used as housekeeping gene. RHEK1 par and RHEK1 par C RNAi, respectively, were used to normalise the data. The nucleic acid intercalating property of the fluorescent dye SYBR-Green (Applied Biosystems, Weiterstadt, Germany) was utilised to measure the extent of gene replication in line with $\Delta \Delta \mathrm{C}_{\mathrm{T}}$ qRT-PCR method with efficiency correction (Fink et al, 1998).

\section{Knockdown of c-myc by siRNA and c-myc/LMP1 transfection}

To knockdown c-myc, cells were reverse transfected with ON-TARGET plus SMART pool (Thermo Scientific, Dharmacon, Bonn, Germany). The following oligos were synthesised by Dharmacon: 5'UUACGCACAAGAGUUCCGU3'; $5^{\prime}$ UCCAAGACGU $^{\prime}$ UGUGUGUUC3 ${ }^{\prime}$; $5^{\prime}$ UGUUGGUGAAGCUAACGUU3'; $^{\prime}$; and $5^{\prime}$ UUCC ACAGAAACAACAUCG3'. The ON-TARGET plus Non-targeting Pool of at least four mismatches to any human gene served as a negative control. siRNAs were transfected using DharmaFECT1 Transfection Reagent (Thermo Scientific). For the (co)transfection of RHEK1 with pLNSX-LMP1 and pcDNA3-c-myc (Ricci et al, 2004) Lipofectamine LTX and PLUS Reagent (Invitrogen) was used. Empty vector controls were included.

\section{Flow cytometric analysis}

RHEK1 cells were stained with HC10 antibody (1:100; Stam et al, 1986; Perosa et al, 2003) for $30 \mathrm{~min}$ at RT. Flow cytometric analysis was performed on a FACSCanto II flow cytometer using the FACSDiva software v6.1.3 (BD, Heidelberg, Germany).

\section{IL6 ELISA}

In all, 96-well plates were coated with an IL6 antibody (MAB206, $\mathrm{R} \& \mathrm{D}$, Minneapolis, MN, USA). After blocking with $1 \%$ BSA, standards and cell supernatants were added for $2 \mathrm{~h}$. After incubation with the detection antibody (BAF206, R\&D), streptavidin-HRP complex (R\&D) and the substrate were added. To stop the staining reaction $0.18 \mathrm{M}$ sulphur dioxide was used. Absorbance was measured at 450 and $590 \mathrm{~nm}$ as reference.

\section{RESULTS}

\section{LMP1 induced up-regulation of HLA class I APM and} c-Myc in epithelial cells

In order to analyse the regulation of the HLA class I APM components by LMP1, the expression of proteasome subunits, the transporter system, chaperones, HLA class I HC (HLA-A and HC10) and $\beta_{2}-\mathrm{m}$ (Supplementary Table S1) was investigated by immunocytochemistry in epithelial cells. Human leukocyte antigen-A, $\beta_{2}-\mathrm{m}$, LMP2 and TAP1 were visibly up-regulated in RHEK1/ LMP1 compared with RHEK1 cells (Figure 1A). No difference was seen in other components. In flow cytometric analysis, HC10 positivity was increased in RHEK1/LMP1 compared with RHEK1 (Figure 1B). For further experiments, HC10, HLA-A, $\beta_{2}-\mathrm{m}, \mathrm{LMP} 2$ and TAP1 were chosen as LMP1 - targets. The effect of LMP1 was confirmed in RHEK1 and HeLa cells in immunoblotting (Figure 1C) and qRT-PCR (data not shown). c-Myc expression decreased strongly with increasing cell confluence (Figure 1D) with highest c-Myc levels around 50\% confluence (used for further experiments). At protein level, LMP1 induced c-Myc expression in RHEK1 and HeLa cells (Figure 1D). At RNA level, significant c-myc induction was only observed in RHEK1/LMP1 cells (Figure 1E).

\section{Opposing effect of c-myc on LMP1 - induced up-regulation of HLA class I APM}

c-myc was knocked down in RHEK1 and HeLa (data not shown) cells using c-myc-specific siRNA and resulted in an increase in HLA class I HC, $\beta_{2}-\mathrm{m}$, TAP1 and LMP2 expression at RNA 
(Figure 2A) and protein level (Figure 2B). The effect was more pronounced in RHEK1/LMP1 with higher baseline levels of c-Myc expression. An increase of superficial HLA class I HC10 was found in RHEK1 cells. This effect was even enhanced in RHEK1/LMP1 compared with RHEK1 (Figure 2C). Thereafter, RHEK1 cells were transiently reverse cotransfected with LMP1, c-myc or both. $\beta_{2}-\mathrm{m}$, TAP1 and LMP2 expression was increased when LMP1 was transfected alone. This effect was diminished when cells were cotransfected with c-myc (Figure 3A). Latent membrane protein 1 expression levels under conditions of sole and cotransfection were similar in qRT-PCR (Figure 3B). In flow cytometric analysis, cotransfection with c-myc mildly decreased the effect of LMP1 on the surface expression of the HLA class I HC (HC10) (Figure 3C).

\section{Induction of STAT3 pathway and IL6 by LMP1}

Western blotting of fractionated cell lysates revealed increased STAT3-pY705 phosphorylation in both the cytosolic and the nuclear fractions of LMP1 expressing cells (Figure 4A). ELISA revealed a strong inductive effect of LMP1 on IL6 secretion (Figure 4B). Moreover, IL6 stimulation induced STAT3 phosphorylation and c-Myc expression (Figure 4C). Finally, treatment of RHEK1/LMP1 cells with the JAK3 inhibitor CP690550 almost completely abolished STAT3-pY705 phosphorylation accompanied by a dose-dependent decrease in c-Myc expression (Figure 4D).

\section{Down-regulation of HLA-A, LMP7, TAP2 and tapasin in NPC and up-regulation of c-Myc and Ki-67 in LMP1 + NPC in immunohistochemistry}

Nasopharyngeal epithelium was compared with NPC cases (LMP1,$- n=9$; LMP1,$+ n=7$ ) using the same set of antibodies investigated in cell culture (Supplementary Table S1; Figure 5A). No significant differences were observed when comparing columnar or squamous epithelium. When comparing NPC and control tissues, a significant down-regulation of LMP7 $(P<0.001)$, TAP1 $(P<0.001)$, tapasin $(P=0.019)$ and HLA-A $(P<0.001)$ was observed in NPC. Only weak expression of LMP2 and TAP1 was detected in NPC and control tissue. No difference in the expression of APM components including HLA-A, HLA-B and HC10-detected HCs was found when comparing LMP1 - and LMP1 + NPC cases. When evaluating the expression of the oncoprotein c-Myc and the proliferation associated antigen Ki-67, a significant increase of both markers was observed in NPC compared with control tissue (c-Myc, $P<0.001$; Ki-67, $P<0.001$ ) (Figure 5B and $\mathrm{C}$ ). Moreover, c-Myc $(P=0.027)$ and Ki-67 $(P=0.027)$ were up-regulated in LMP1 + NPC compared with LMP1 - NPC cases. To exclude that the lack of difference between LMP1 - and LMP1 + NPC cases was a consequence of localised or focal expression of LMP1, serial sections of three LMP1 + NPC were stained for LMP1 and three APM components regulated by LMP1. The expression of LMP1 was patchy or focal in only few scattered cells in all three cases, whereas the expression of $\beta_{2}-\mathrm{m}$, HLA-A and LMP2 showed a diffuse and homogenous distribution (Figure 5D).

\section{DISCUSSION}

Down-regulation of the HLA class I APM is a mechanism used by malignancies to elicit immune evasion (Chang et al, 2003). Therefore, it appears paradoxical that the EBV oncogene LMP1 induces HLA class I APM components. Here, we found that stable transfection of epithelial cells with LMP1 lead to a strong upregulation of the HLA class I APM as has been reported for B cells (Rowe et al, 1995). At the same time, LMP1 induced c-Myc, which has been shown to down-regulate HLA class I APM expression (Versteeg et al, 1988; Ottesen et al, 1990; Belldegrun et al, 1993; Staege et al, 2002) inducing a non-immunogenic phenotype.
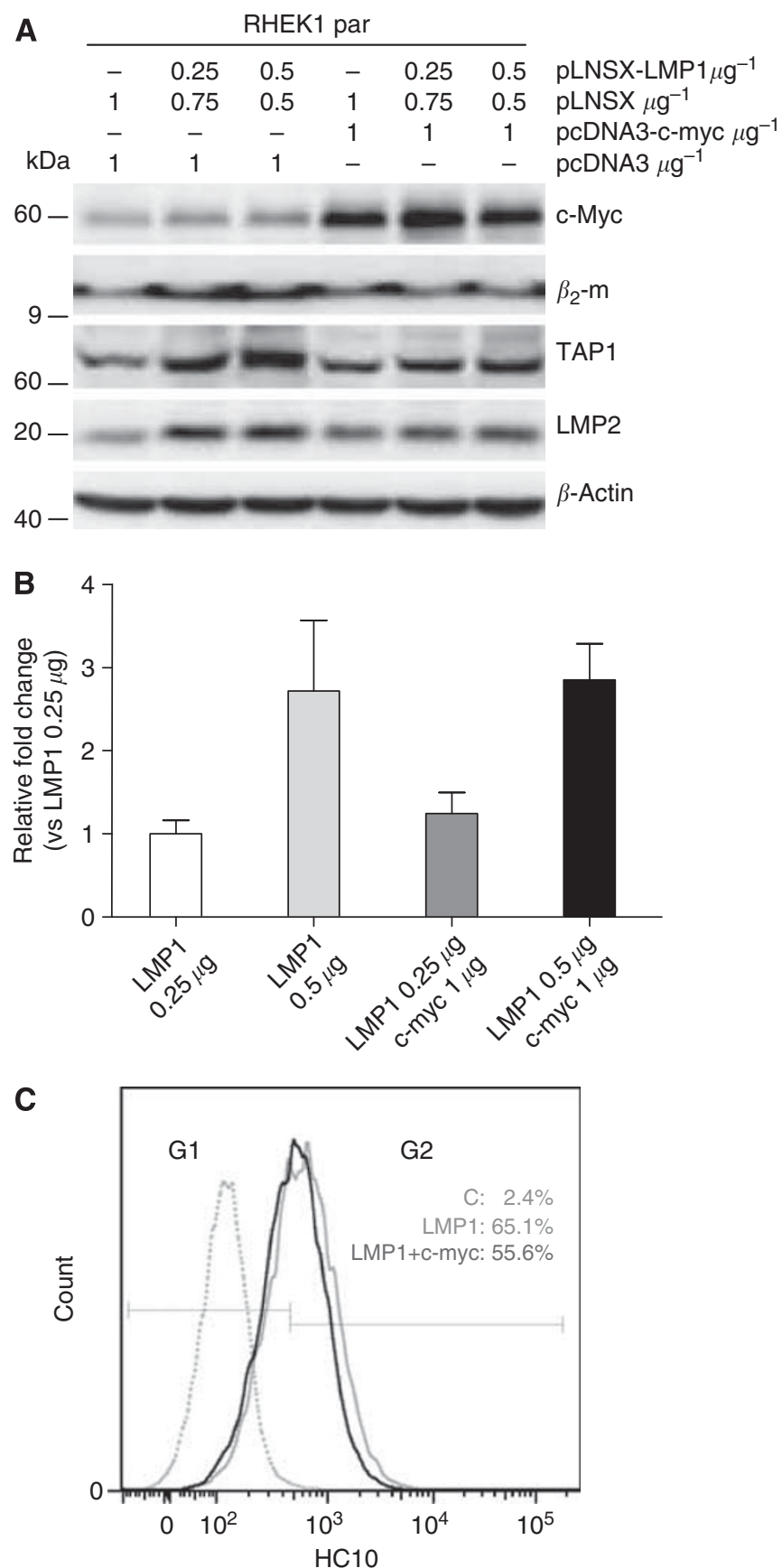

Figure 3 Inhibitory effect of c-myc on LMPI-mediated induction of HLA class I APM components in cotransfection experiments. (A) Western blot analysis showing expression of $\beta_{2}-\mathrm{m}$, TAPI and LMP2 after transient transfection with LMPI or c-myc alone or after cotransfection with LMPI and c-myc. As shown in the stably transfected cell lines, transient transfection with LMPI leads to an induction of $\beta_{2}-\mathrm{m}$, TAPI and LMP2. The inductive effect of LMPI is reduced when cotransfected with c-myc. One representative experiment of 3 . (B) Comparison of LMPI levels after transfection with LMPI alone or after cotransfection with c-myc by qRT-PCR. Comparative levels of LMPI were seen in the single transfection and cotransfection. One representative experiment of 3. (C) Flow cytometric anaylsis of RHEKI parental comparing the transient transfection of LMPI $(0.5 \mu \mathrm{g})$ alone and the cotransfection of LMPI $(0.5 \mu \mathrm{g})$ and c-myc (I $\mu \mathrm{g})$. A significant inhibitory effect $(P=0.049 ; n=5)$ of $c-m y c$ on the inductive effect of LMPI on HLA class I HC $(\mathrm{HClO})$ is seen. The dotted line represents the control staining without primary antibody. The solid light/dark line shows $\mathrm{HClO}$ expression in transiently LMPI/c-myc and LMPI cotransfected RHEKI cells. The percentage of positive cells in gate G2 is indicated in the control (C), in LMPI-transfected cells (LMPI) and in LMPI and c-myc cotransfected cells (LMPI + c-myc) for the representative experiment of 5. 
A

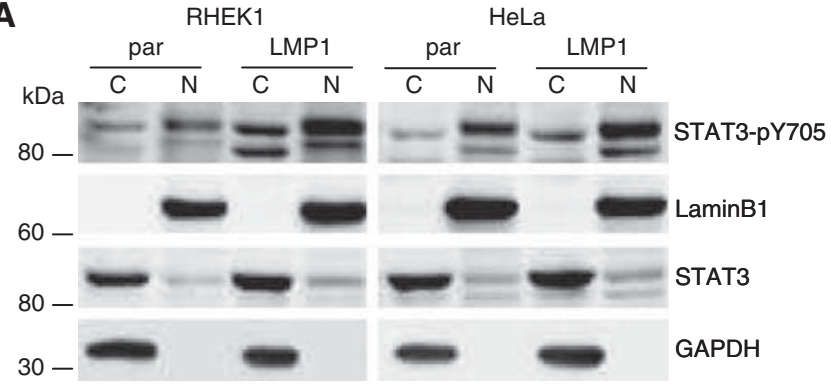

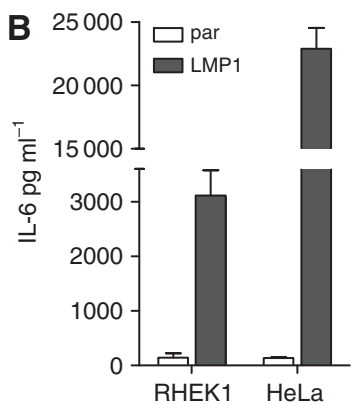
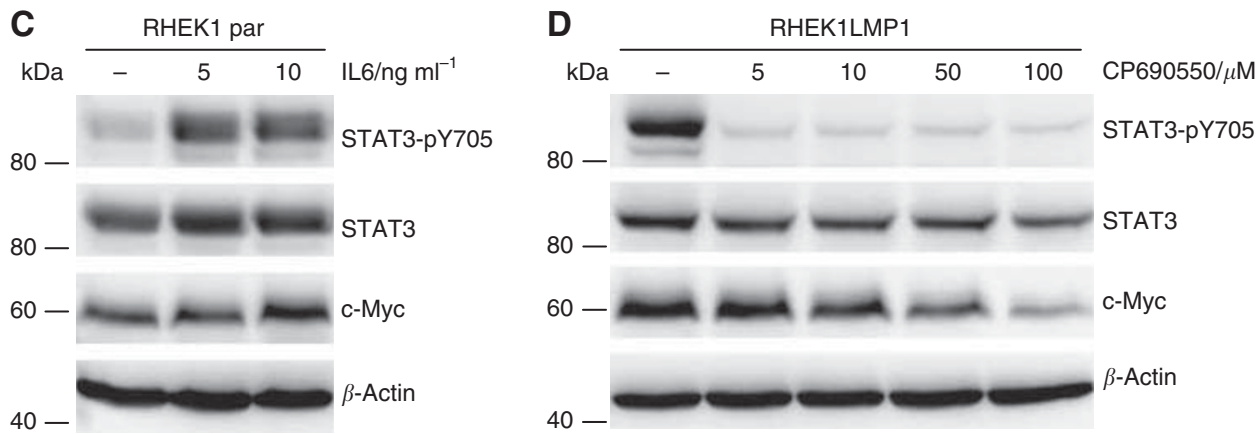

Figure 4 Interleukin-6 and STAT3 as mediators of the c-Myc regulation in epithelial cells. (A) Western blot analysis of the expression of STAT3 and STAT3-pY705 phosphorylation status in nuclear and cytoplasmic fractions of RHEKI and HeLa cells depending on the presence of LMPI. Stable transfection with LMPI leads to a mild induction of cytoplasmic STAT3 and to a pronounced induction of cytosolic and nuclear STAT3-pY705 phosporylation. One representative experiment of 3. (B) ELISA analysis of the effect of stable LMPI transfection on the expression of IL6. A strong induction of secreted IL6 is observed in both RHEKI and HeLa cells. Mean values and standard deviations of three independent experiments. (C) Western blot analysis of the effect of recombinant human IL6 on STAT3, STAT3-pY705 phosphorylation and c-Myc in RHEK parental. IL6 leads to a mild up-regulation of STAT3, c-ymc and a strong induction of STAT3-pY705 phosphorylation. One representative experiment of 3. (D) Western blot analysis showing the effect of the JAK3 inhibitor CP690550 on STAT3, STAT3-pY705 phosphorylation and c-Myc. Incubation of RHEKI/LMPI with CP690550 for $48 \mathrm{~h}$ leads to strong reduction of STAT3pY705 phosphorylation and to a reduction in STAT3 and c-Myc expression in a dose-dependent manner. One representative experiment of 3.

Therefore, c-Myc was a candidate to counteract the immunogenicity of LMP1. Indeed, c-Myc had an opposing effect on HLA class I APM expression seen as an up-regulation of the HLA class I APM after c-myc knockdown and an inhibition of the inductive effect of LMP1, when LMP1 and c-myc were coexpressed. Additionally, we found that STAT3 might mediate this effect, as LMP1 strongly up-regulated STAT3 phosphorylation. STAT3 activation can be achieved directly via JAK3 activation (Gires et al, 1999) or via secreted mediators in an autocrine or paracrine manner. Interleukin-6 is thought to be involved in a positive feedback loop of LMP1 expression and STAT3 activation (Chen et al, 2003). Moreover, IL6 is found in a subset of EBV-associated NPC cases in tumour cells and/or the infiltrate (Okamoto et al, 1997; Hirano et al, 2000; Beck et al, 2001; Lesina et al, 2011). We showed that LMP1 induced IL6 secretion in agreement with earlier reports (Eliopoulos et al, 1997) and that IL6 induced c-Myc expression and STAT3 activation. Whereas JAK2 inhibitors only weakly inhibited STAT3 phosphorylation (data not shown), the JAK3 inhibitor CP690550 had a strong negative effect on STAT3 phosphorylation and, in a dose-dependent manner, on c-Myc expression, indicating an important role of JAK3/STAT3 in c-myc regulation in this context. This is supported by an earlier observation showing that JAK3 is directly activated by LMP1 leading to STAT3 phosphorylation (Gires et al, 1999).

Taken together, we propose a model in which LMP1 induces the HLA class I APM in epithelial cells and at the same time opposes this effect by activating c-Myc via JAK3 and STAT3, which in turn down-regulates HLA class I APM. In this scenario, LMP1 could counteract its own immunogenicity.

To test the relevance of these findings in vivo, we investigated the expression of HLA class I APM components and of c-Myc in a small number of NPC biopsies. Previous studies examining the expression of HLA class I APM in NPC have produced conflicting results. While normal levels of LMP2, LMP7, TAP1, TAP2 and HLA class I alleles on NPC tumour cells have been described in one study (Khanna et al, 1998), others have shown heterogeneous expression with a partial loss of HLA-ABC in almost $50 \%$ of NPC cases (Lai et al, 1990). A genome-wide expression profiling study identified a positive correlation between EBV status and reduced MHC class I expression in NPC (Sengupta et al, 2006). Ogino et al (2007) found a down-regulation of HLA class I HC, tapasin, LMP2 and TAP1 in NPC, although no association was observed with respect to LMP1 status. Consistently, we detected down-regulation of tapasin in NPC cases. Additionally, we found a selective down-regulation of HLA-A and no evidence of HLA-B reduction as has been observed in Burkitt lymphoma (Masucci et al, 1987). Demanet et al (2004) reported a selective down-regulation of HLA-A and HLA-Bw6, but not of HLA-Bw4 and suggested that this effect might help to evade CTL and NK cells simultaneously, as HLA-Bw4 can inhibit NK cell function. Hypothetically, a similar effect using divergent functions of different HLA class I alleles in immune escape might be operational in NPC explaining the observed differential regulation of HLA class I subtypes.

The TAP2 and LMP7 were found to be down-regulated in NPC, which were either not investigated (Ogino et al, 2007) or unaltered in earlier studies (Khanna et al, 1998). In the latter study, lymphoblastoid cell lines served as controls compared with nasopharyngeal epithelium in our study, which might explain some of the difference. In contrast to previous findings (Ogino et al, 2007), TAP1 and LMP2 were not down-regulated but showed a low expression level in tumours and control tissues. The concomitant low levels of TAP1 and LMP2 could be explained by the regulation of both genes by the same bidirectional promoter (Wright et al, 1995). In agreement with Ogino et al, we observed no association between LMP1 and HLA class I APM expression, which contrasts with the findings in EBV-positive Hodgkin's 
A

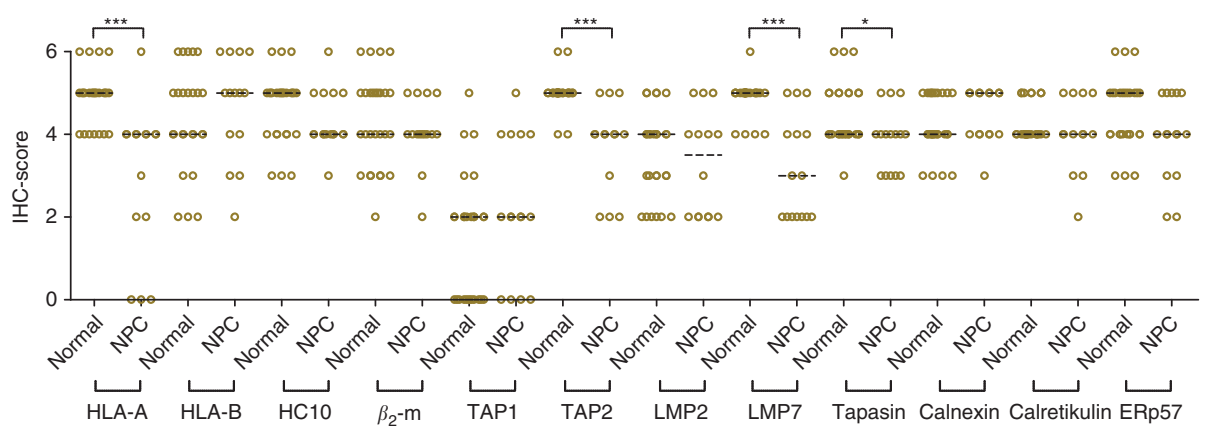

B
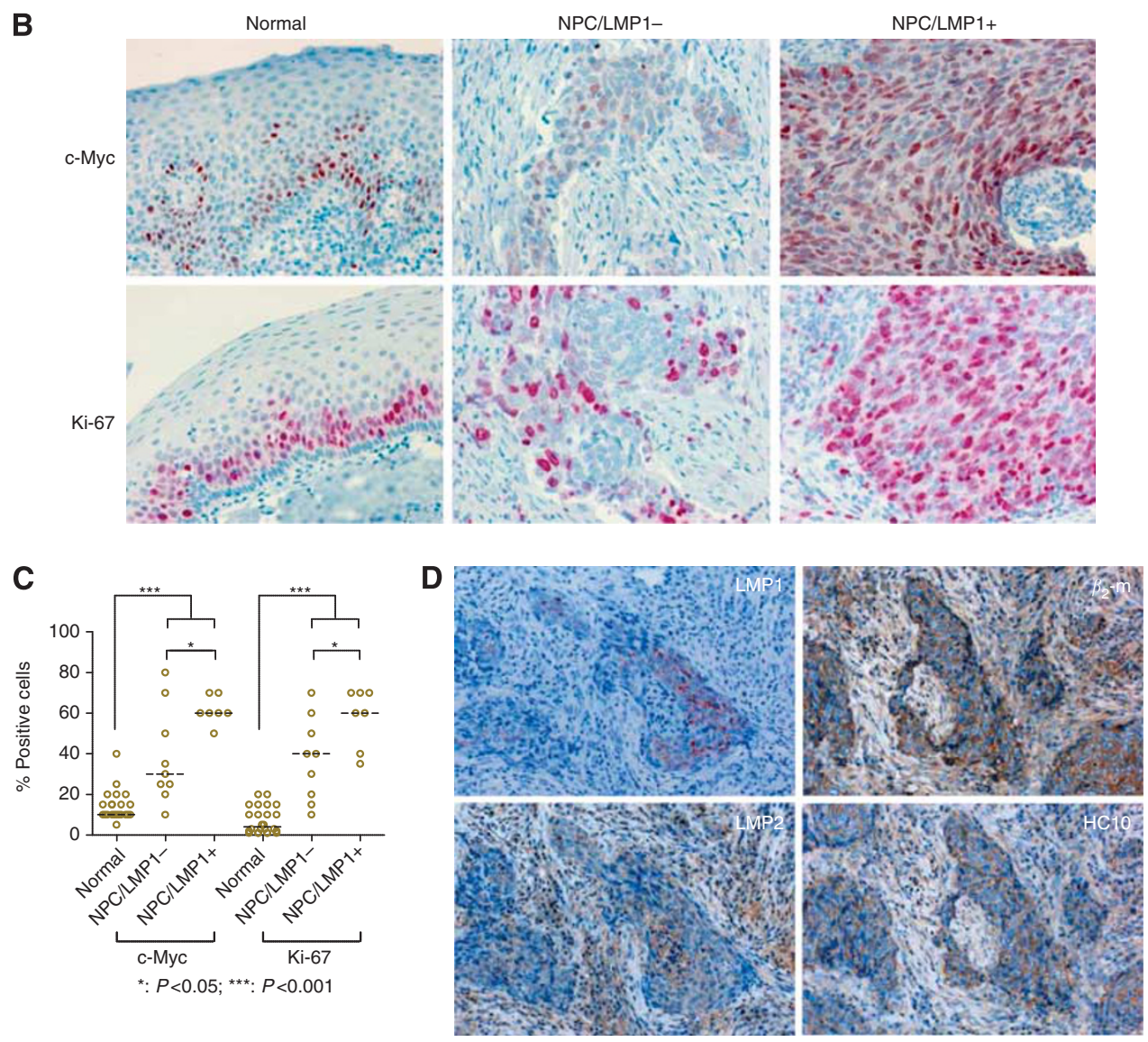

Figure 5 Down-regulation of HLA class I APM components in NPC and up-regulation of c-Myc in LMPI + NPC. (A) Immunohistochemical scores of HLA class I APM components comparing normal tissue (columnar and squamous epithelium) with all NPC cases. HLA-A, TAP2, LMP7 and tapasin were down-regulated in NPC compared with control tissues. Expression of TAPI and LMP2 was low but comparable in both NPC and controls. The median is indicated with a black dotted line. (B) c-Myc and Ki-67 immunohistochemistry of normal nasopharyngeal epithelium and one LMPI - NPC showing a low expression of both markers compared with one LMPI + NPC with high expression of c-Myc and Ki-67. Magnification $\times 400$. $(\mathbf{C})$ Immunohistochemical comparison of the nuclear expression of Ki-67 and c-Myc shown as the percentage of positive nuclei. All normal tissues are compared with LMPI + and LMPI - NPC. Ki-67 and c-Myc are up-regulated in all NPC compared with controls and in LMPI + compared with LMPI - NPC. The median is indicated with a black dotted line. (D) Serial sections of one LMPI + NPC showing a patchy staining of LMPI, whereas the stainings for $\beta_{2}$-m, LMP2 and HLA class I $\mathrm{HC}(\mathrm{HClO})$ are diffuse. Numerous inflammatory cells in the background express the components of the HLA class I APM. Magnification $\times 400$.

disease, where LMP1 is expressed at high levels (Murray et al, 1998).

As LMP1 is usually expressed focally in NPC, we set out to determine whether the lack of an LMP1 - specific effect was a consequence of this. In serial sections of NPC, we indeed found a focal/patchy pattern of LMP1 expression. Expression of HLA class I APM components, however, was diffuse. Therefore, we concluded that any possible effect of LMP1 on the HLA class I APM was not focal maybe due to paracrine effects (e.g., IL6) of LMP1 or the activation of CD40 expressed on LMP1- NPC cells
(Agathanggelou et al, 1995) by surrounding inflammatory cells. Going in line with the in-vitro findings, LMP1 + NPC showed increased expression of c-Myc compared with negative cases. This might explain the failure of LMP1 to induce HLA class I APM expression. The histological findings, however, have some limitation because of the small number of investigated NPC cases. However, an earlier observation that STAT3 phosphorylation in NPC biopsies was independent of LMP1 (Buettner et al, 2006) indicates that the interacting signalling pathways in NPC are more complex than in the in-vitro system. Moreover, the 
phosphorylation of STAT3 might also be transient, so that differences could not be detected by immunohistochemistry.

This model will not explain the entire mechanisms involved in HLA class I APM regulation in NPC as many other factors like the surface expression of CD40 in NPC and the expression of cytokines in the surrounding inflammatory cells will interact with this regulatory circuit. However, it may explain in part the lack of a difference in HLA class I APM expression in LMP1 + and LMP1 NPC. We speculate that overexpression of c-Myc by LMP1 may counteract or reduce the effects of LMP1 on HLA class I APM expression in epithelial cells.

\section{REFERENCES}

Agathanggelou A, Niedobitek G, Chen R, Nicholls J, Yin W, Young LS (1995) Expression of immune regulatory molecules in Epstein-Barr virus-associated nasopharyngeal carcinomas with prominent lymphoid stroma. Evidence for a functional interaction between epithelial tumor cells and infiltrating lymphoid cells. Am J Pathol 147(4): 1152-1160

Bandoh N, Ogino T, Cho HS, Hur SY, Shen J, Wang X, Kato S, Miyokawa N, Harabuchi Y, Ferrone S (2005) Development and characterization of human constitutive proteasome and immunoproteasome subunitspecific monoclonal antibodies. Tissue Antigens 66(3): 185-194

Beck A, Pazolt D, Grabenbauer GG, Nicholls JM, Herbst H, Young LS, Niedobitek G (2001) Expression of cytokine and chemokine genes in Epstein-Barr virus-associated nasopharyngeal carcinoma: comparison with Hodgkin's disease. I Pathol 194(2): 145-151

Belldegrun A, Tso CL, Sakata T, Duckett T, Brunda MJ, Barsky SH, Chai J, Kaboo R, Lavey RS, McBride WH, deKernion JB (1993) Human renal carcinoma line transfected with interleukin-2 and/or interferon alpha gene(s): implications for live cancer vaccines. J Natl Cancer Inst 85(3): 207-216

Blom DJ, Mooy CM, Luyten GP, Kerkvliet S, Ouwerkerk I, Zwinderman AH, Schrier PI, Jager MJ (1997) Inverse correlation between expression of HLA-B and c-myc in uveal melanoma. $J$ Pathol 181(1): 75-79

Bowman T, Broome MA, Sinibaldi D, Wharton W, Pledger WJ, Sedivy JM, Irby R, Yeatman T, Courtneidge SA, Jove R (2001) Stat3-mediated Myc expression is required for Src transformation and PDGF-induced mitogenesis. Proc Natl Acad Sci USA 98(13): 7319-7324

Bromberg JF, Wrzeszczynska MH, Devgan G, Zhao Y, Pestell RG, Albanese C, Darnell Jr JE (1999) Stat3 as an oncogene. Cell 98(3): 295-303

Buettner M, Heussinger N, Niedobitek G (2006) Expression of Epstein-Barr virus (EBV)-encoded latent membrane proteins and STAT3 activation in nasopharyngeal carcinoma. Virchows Arch 449(5): 513-519

Chang CC, Campoli M, Ferrone S (2003) HLA class I defects in malignant lesions: what have we learned? Keio J Med 52(4): 220-229

Chen H, Hutt-Fletcher L, Cao L, Hayward SD (2003) A positive autoregulatory loop of LMP1 expression and STAT activation in epithelial cells latently infected with Epstein-Barr virus. J Virol 77(7): 4139-4148

Dahl E, Wiesmann F, Woenckhaus M, Stoehr R, Wild PJ, Veeck J, Knuchel R, Klopocki E, Sauter G, Simon R, Wieland WF, Walter B, Denzinger S, Hartmann A, Hammerschmied CG (2007) Frequent loss of SFRP1 expression in multiple human solid tumours: association with aberrant promoter methylation in renal cell carcinoma. Oncogene 26(38): 5680-5691

Dawson CW, Laverick L, Morris MA, Tramoutanis G, Young LS (2008) Epstein-Barr virus-encoded LMP1 regulates epithelial cell motility and invasion via the ERK-MAPK pathway. J Virol 82(7): 3654-3664

Demanet C, Mulder A, Deneys V, Worsham MJ, Maes P, Claas FH, Ferrone S (2004) Down-regulation of HLA-A and HLA-Bw6, but not HLA-Bw4, allospecificities in leukemic cells: an escape mechanism from CTL and NK attack? Blood 103(8): 3122-3130

Diosdado B, van de Wiel MA, Terhaar Sive Droste JS, Mongera S, Postma C, Meijerink WJ, Carvalho B, Meijer GA (2009) MiR-17-92 cluster is associated with $13 \mathrm{q}$ gain and $\mathrm{c}$-myc expression during colorectal adenoma to adenocarcinoma progression. Br J Cancer 101(4): 707-714

Eliopoulos AG, Blake SM, Floettmann JE, Rowe M, Young LS (1999) Epstein-Barr virus-encoded latent membrane protein 1 activates the JNK pathway through its extreme $\mathrm{C}$ terminus via a mechanism involving TRADD and TRAF2. J Virol 73(2): 1023-1035

Eliopoulos AG, Stack M, Dawson CW, Kaye KM, Hodgkin L, Sihota S, Rowe M, Young LS (1997) Epstein-Barr virus-encoded LMP1 and CD40

\section{ACKNOWLEDGEMENTS}

We thank Reinhard Voll for critical discussion and valuable advice and Birgit Meyer for excellent technical assistance. MS Ricci was the principal investigator to generate the c-myc plasmid. This work was supported by the Graduiertenkolleg (GRK) 1071, Viruses of the Immune System, Erlangen, Germany.

Supplementary Information accompanies the paper on British Journal of Cancer website (http://www.nature.com/bjc)

mediate IL-6 production in epithelial cells via an NF-kappaB pathway involving TNF receptor-associated factors. Oncogene 14(24): 2899-2916

Fahraeus R, Fu HL, Ernberg I, Finke J, Rowe M, Klein G, Falk K, Nilsson E, Yadav M, Busson P, Tursz T, Kallin B (1988) Expression of Epstein-Barr virus-encoded proteins in nasopharyngeal carcinoma. Int J Cancer 42(3): $329-338$

Fink L, Seeger W, Ermert L, Hanze J, Stahl U, Grimminger F, Kummer W, Bohle RM (1998) Real-time quantitative RT-PCR after laser-assisted cell picking. Nat Med 4(11): 1329-1333

Flutter B, Gao B (2004) MHC class I antigen presentation-recently trimmed and well presented. Cell Mol Immunol 1(1): 22-30

Gires O, Kohlhuber F, Kilger E, Baumann M, Kieser A, Kaiser C, Zeidler R, Scheffer B, Ueffing M, Hammerschmidt W (1999) Latent membrane protein 1 of Epstein-Barr virus interacts with JAK3 and activates STAT proteins. EMBO J 18(11): 3064-3073

Greiner A, Tobollik S, Buettner M, Jungnickel B, Herrmann K, Kremmer E, Niedobitek G (2005) Differential expression of activation-induced cytidine deaminase (AID) in nodular lymphocyte-predominant and classical Hodgkin lymphoma. J Pathol 205(5): 541-547

Heussinger N, Buttner M, Ott G, Brachtel E, Pilch BZ, Kremmer E, Niedobitek G (2004) Expression of the Epstein-Barr virus (EBV)-encoded latent membrane protein 2A (LMP2A) in EBV-associated nasopharyngeal carcinoma. J Pathol 203(2): 696-699

Hirano T, Ishihara K, Hibi M (2000) Roles of STAT3 in mediating the cell growth, differentiation and survival signals relayed through the IL-6 family of cytokine receptors. Oncogene 19(21): 2548-2556

Kasper G, Weiser AA, Rump A, Sparbier K, Dahl E, Hartmann A, Wild P, Schwidetzky U, Castanos-Velez E, Lehmann K (2005) Expression levels of the putative zinc transporter LIV-1 are associated with a better outcome of breast cancer patients. Int J Cancer 117(6): 961-973

Khanna R, Busson P, Burrows SR, Raffoux C, Moss DJ, Nicholls JM, Cooper L (1998) Molecular characterization of antigen-processing function in nasopharyngeal carcinoma (NPC): evidence for efficient presentation of Epstein-Barr virus cytotoxic T-cell epitopes by NPC cells. Cancer Res 58(2): 310-314

Lai FM, Cheng PN, Tsao SY, Lai KN (1990) Immunohistological characteristics of the infiltrating lymphoid cells and expression of HLA class I and II antigens in nasopharyngeal carcinoma. Virchows Arch A Pathol Anat Histopathol 417(4): 347-352

Lampson LA, Fisher CA, Whelan JP (1983) Striking paucity of HLA-A, B, C and beta 2-microglobulin on human neuroblastoma cell lines. J Immunol 130(5): 2471-2478

Lesina M, Kurkowski MU, Ludes K, Rose-John S, Treiber M, Kloppel G, Yoshimura A, Reindl W, Sipos B, Akira S, Schmid RM, Algul H (2011) Stat3/Socs 3 activation by IL-6 transsignaling promotes progression of pancreatic intraepithelial neoplasia and development of pancreatic cancer. Cancer Cell 19(4): 456-469

Lin SY, Tsang NM, Kao SC, Hsieh YL, Chen YP, Tsai CS, Kuo TT, Hao SP, Chen IH, Hong JH (2001) Presence of Epstein-Barr virus latent membrane protein 1 gene in the nasopharyngeal swabs from patients with nasopharyngeal carcinoma. Head Neck 23(3): 194-200

Masucci MG, Torsteindottir S, Colombani J, Brautbar C, Klein E, Klein G (1987) Down-regulation of class I HLA antigens and of the Epstein-Barr virus-encoded latent membrane protein in Burkitt lymphoma lines. Proc Natl Acad Sci USA 84(13): 4567-4571

Murray PG, Constandinou CM, Crocker J, Young LS, Ambinder RF (1998) Analysis of major histocompatibility complex class I, TAP expression, 
and LMP2 epitope sequence in Epstein-Barr virus-positive Hodgkin's disease. Blood 92(7): 2477-2483

Niedobitek G, Herbst H (2001) In situ detection of Epstein-Barr virus DNA and viral gene products. Methods Mol Biol 174: 79-91

Niedobitek G, Young LS, Sam CK, Brooks L, Prasad U, Rickinson AB (1992) Expression of Epstein-Barr virus genes and of lymphocyte activation molecules in undifferentiated nasopharyngeal carcinomas. Am J Pathol 140(4): 879-887

Ogino T, Moriai S, Ishida Y, Ishii H, Katayama A, Miyokawa N, Harabuchi Y, Ferrone S (2007) Association of immunoescape mechanisms with Epstein-Barr virus infection in nasopharyngeal carcinoma. Int J Cancer 120(11): 2401-2410

Ogino T, Wang X, Kato S, Miyokawa N, Harabuchi Y, Ferrone S (2003) Endoplasmic reticulum chaperone-specific monoclonal antibodies for flow cytometry and immunohistochemical staining. Tissue Antigens 62(5): 385-393

Okamoto M, Lee C, Oyasu R (1997) Interleukin-6 as a paracrine and autocrine growth factor in human prostatic carcinoma cells in vitro. Cancer Res 57(1): 141-146

Ottesen SS, Skouv J, Kieler J (1990) Reduced HLA-A,B,C expression in tumourigenic v-raf transfected human urothelial cells. Eur J Cancer 26(3): 305-310

Perosa F, Luccarelli G, Prete M, Favoino E, Ferrone S, Dammacco F (2003) Beta 2-microglobulin-free HLA class I heavy chain epitope mimicry by monoclonal antibody HC-10-specific peptide. J Immunol 171(4): 1918-1926

Ravetch JV, Lanier LL (2000) Immune inhibitory receptors. Science 290(5489): 84-89

Respa A, Bukur J, Ferrone S, Pawelec G, Zhao Y, Wang E, Marincola FM, Seliger B (2011) Association of IFN-gamma signal transduction defects with impaired HLA class I antigen processing in melanoma cell lines. Clin Cancer Res 17(9): 2668-2678

Ricci MS, Jin Z, Dews M, Yu D, Thomas-Tikhonenko A, Dicker DT El-Deiry WS (2004) Direct repression of FLIP expression by c-myc is a major determinant of TRAIL sensitivity. Mol Cell Biol 24(19): 8541-8555

Rowe M, Khanna R, Jacob CA, Argaet V, Kelly A, Powis S, Belich M, Croom-Carter D, Lee S, Burrows SR, Trowsdale J, Moss DJ, Rickinson AB (1995) Restoration of endogenous antigen processing in Burkitt's lymphoma cells by Epstein-Barr virus latent membrane protein-1: coordinate up-regulation of peptide transporters and HLA-class I antigen expression. Eur J Immunol 25(5): 1374-1384

Seliger B, Cabrera T, Garrido F, Ferrone S (2002) HLA class I antigen abnormalities and immune escape by malignant cells. Semin Cancer Biol 12(1): 3-13

Seliger B, Stoehr R, Handke D, Mueller A, Ferrone S, Wullich B Tannapfel A, Hofstaedter F, Hartmann A (2010) Association of HLA class I antigen abnormalities with disease progression and early recurrence in prostate cancer. Cancer Immunol Immunother 59(4): $529-540$
Sengupta S, den Boon JA, Chen IH, Newton MA, Dahl DB, Chen M, Cheng YJ, Westra WH, Chen CJ, Hildesheim A, Sugden B, Ahlquist P (2006) Genome-wide expression profiling reveals EBV-associated inhibition of MHC class I expression in nasopharyngeal carcinoma. Cancer Res 66(16): 7999-8006

Sernee MF, Ploegh HL, Schust DJ (1998) Why certain antibodies cross-react with HLA-A and HLA-G: epitope mapping of two common MHC class I reagents. Mol Immunol 35(3): 177-188

Siegler G, Meyer B, Dawson C, Brachtel E, Lennerz J, Koch C, Kremmer E, Niedobitek E, Gonnella R, Pilch BZ, Young LS, Niedobitek G (2004) Expression of tumor necrosis factor receptor-associated factor 1 in nasopharyngeal carcinoma: possible upregulation by Epstein-Barr virus latent membrane protein 1. Int J Cancer 112(2): 265-272

Staege MS, Lee SP, Frisan T, Mautner J, Scholz S, Pajic A, Rickinson AB, Masucci MG, Polack A, Bornkamm GW (2002) MYC overexpression imposes a nonimmunogenic phenotype on Epstein-Barr virus-infected B cells. Proc Natl Acad Sci USA 99(7): 4550-4555

Stam NJ, Spits H, Ploegh HL (1986) Monoclonal antibodies raised against denatured HLA-B locus heavy chains permit biochemical characterization of certain HLA-C locus products. J Immunol 137(7): 2299-2306

Uchida J, Yasui T, Takaoka-Shichijo Y, Muraoka M, Kulwichit W, Raab-Traub N, Kikutani H (1999) Mimicry of CD40 signals by Epstein-Barr virus LMP1 in B lymphocyte responses. Science 286(5438): 300-303

van Oers JM, Wild PJ, Burger M, Denzinger S, Stoehr R, Rosskopf E, Hofstaedter F, Steyerberg EW, Klinkhammer-Schalke M, Zwarthoff EC van der Kwast TH, Hartmann A (2007) FGFR3 mutations and a normal CK20 staining pattern define low-grade noninvasive urothelial bladder tumours. Eur Urol 52(3): 760-768

Versteeg R, Noordermeer IA, Kruse-Wolters M, Ruiter DJ, Schrier PI (1988) c-myc down-regulates class I HLA expression in human melanomas. EMBO J 7(4): 1023-1029

Vitale M, Pelusi G, Taroni B, Gobbi G, Micheloni C, Rezzani R, Donato F, Wang X, Ferrone S (2005) HLA class I antigen down-regulation in primary ovary carcinoma lesions: association with disease stage. Clin Cancer Res 11(1): 67-72

Wang X, Campoli M, Cho HS, Ogino T, Bandoh N, Shen J, Hur SY, Kageshita T, Ferrone S (2005) A method to generate antigen-specific $\mathrm{mAb}$ capable of staining formalin-fixed, paraffin-embedded tissue sections. J Immunol Methods 299(1-2): 139-151

Wright KL, White LC, Kelly A, Beck S, Trowsdale J, Ting JP (1995) Coordinate regulation of the human TAP1 and LMP2 genes from a shared bidirectional promoter. J Exp Med 181(4): 1459-1471

Xu J, Ahmad A, D'Addario M, Knafo L, Jones JF, Prasad U, Dolcetti R, Vaccher E, Menezes J (2000) Analysis and significance of anti-latent membrane protein-1 antibodies in the sera of patients with EBV-associated diseases. J Immunol 164(5): 2815-2822

Young LS, Rickinson AB (2004) Epstein-Barr virus: 40 years on. Nat Rev Cancer 4(10): 757-768

This work is published under the standard license to publish agreement. After 12 months the work will become freely available and the license terms will switch to a Creative Commons Attribution-NonCommercial-Share Alike 3.0 Unported License. 